\title{
Mosquito control methods and their limitations
}

\author{
Afeefa Chaudhry ${ }^{1,2}$, Rasheda Jabeen ${ }^{1}$, Beenish Sarfraz ${ }^{1}$ and Sumaira \\ Mazhar $^{1 *}$ \\ 1. Department of Biology, Lahore Garrison University, Lahore-Pakistan \\ 2. Center of excellence in molecular biology, Punjab University, Lahore-Pakistan \\ *Corresponding author's email:smz.mmg@gmail.com \\ Citation \\ Afeefa Chaudhry, Rasheda Jabeen, Beenish Sarfraz and Sumaira Mazhar. Mosquito control methods and their \\ limitations. Pure and Applied Biology. Vol. 8, Issue 4, pp2389-2398. http://dx.doi.org/10.19045/bspab.2019.80184

\begin{tabular}{llll}
\hline \hline Received: 06/05/2019 & Revised: 13/07/2019 & Accepted: 23/08/2019 & Online First: 07/09/2019 \\
\hline \hline
\end{tabular}

Abstract

Mosquitoes represent the major vectors involved in the spread of deadly diseases like Malaria, Lymphatic filariasis, Chikungunyaand arboviruses like dengue virus and Zika virus. These diseases have emerged with the rise of urbanization and the use of chemical insecticides around the world. The insecticides such as DDT (Dichlorodiphenyltrichloroethane), organophosphates, carbamates, and organochlorineshave caused resistance in mosquitoes against typical chemical control methodsthrough excessive use over the years. Different biological control methods aresustainable, eco-friendly and target major diseases spreading mosquito's species. This review outlines major biological control methods being used ortested in different parts of the world. Some of these are the use of plant extract, Wolbachiaspp, larvivorous fishes, insecticidal bacterial spp, predator mosquitoes and predator copepods.Limitations associated with each method are also discussed.All these methods will givea better understanding of developing an integrated approach for effective mosquitoes control that will reduce dependency on insecticide based approaches.

Keywords: Biological control; Dengue; DDT; Larvivorous fish; Malaria, Mosquitoes, Wolbachia Introduction

Biotechnological applications of commercially applicable microorganism have led to recent advancements in the fields of agriculture, food and dairy and specifically in the human health sector. Many commercially important microbial species are being used for enhancing the agricultural yield of economically important crops by using the insecticidal and pesticidal activity of certain microbial species without affecting the natural diversity of the ecosystem. Although microbes have proved their significance in agriculture field by increasing crop yield of disease susceptible crops their application concerning human health is still an area of ongoing research. There are many fatal and pandemic diseases which are associated with various insects and pests among which the mosquitoes are playing an important role in the transmission of various diseases. Mosquitoes like Aedes aegypti, Aedes albopictus and Anopheles species have been reported as a transmission source of various diseases like Dengue Fever, Yellow Fever, Chikungunya, Zika virus, Lymphatic filariasis, Malaria and West Nile virus.it has been reported that around $40 \%$ of the whole world's population is at risk of getting the Dengue Fever alone [1]. 
The statistics show that there are more than 627,000 deaths annually reported from all over the world due to Malaria with more than 150 million infections of malaria. More than 100 million infections of Dengue virus are reported each year from all over the world and around $2.5 \%$ of which die due to the disease. has caused more than Around 1.4 million cases ofanother mosquito-borne disease Chikungunya was reported during its worst outbreak in South India in the year 2006. Yellow fever has been reported to cause over 30,000 deaths annually. Japanese Encephalitis is responsible for the death of 10,000 patients annually. There are 120 million cases of Lymphatic filariasis reported from all over the world and 40 million of these cases ended up having impaired body functions or permanent organ failures. All these diseases are transmitted by various life stages of differed mosquito species [2].

Methods of controlling mosquitoes Chemical control of mosquitoes

To control the major genera of mosquitoes such as Anopheles, Aedes, and Culex many chemical insecticides are being used for many years. These chemical insecticides have also contributed much in the field of agriculture by controlling the harmful pests and insects that can alter the net productivity of a specific crop. The major classes of insecticides that have been widely used include carbamates, organophosphates, pyrethroids, and organochlorines. These insecticidesare used forcontrolling several species of mosquitoes that are involved in the transmission of various vector-borne diseases such as Dengue Fever, Malaria, Japanese encephalitis and lymphatic filariasis [3].

Recent studies of insecticidal activity of various classes of chemical insecticides on different life stages of mosquitoes have shown the incidence of resistance developed by major disease-transmitting species such as Aedesaegypti. The xenobiotics also play a major role in inducing genetic variations among populations of mosquitoes which are responsible for inducing different resistance mechanisms such as the exposure of mosquito larvae to fluoranthene and copper increased the level of resistance to permethrin the most widely used insecticide against mosquitoes [4]. It has been observed that the incidence of increasing resistance in different mosquitoes is directly associated with increased urbanization. It has been estimated that by 2050 , more than $70 \%$ of the world's population would start living in the cities and most important and dangerous arthropods that have developed resistance against chemical insecticides belong to urban ecosystems such as Aedes egypti, the vector of Dengue Fever and Yellow Fever and Culex pipiens, the vector of Lymphatic filariasis and Japanese encephalitis.

\section{Mechanism of action of chemical} insecticides

Among all the classes of chemical insecticides after DDT (dichlorodiphenyltrichloroethane) the most widely used chemical insecticide is pyrethroid. A pyrethroid is the only chemical insecticide which has been recommended for use in Insecticide-treated nets (ITNs) and Long-lasting insecticide-treated beds while other classes of insecticides such as organochlorines, organophosphates, and carbamates have been recommended for use as indoor residual sprays (IRS) [5]. These chemical insecticides share specific target site in the cells which are called Voltagegated sodium channels (VGSC). These channels serve as the targets of various other neurotoxins. These channels are transmembrane proteins that function in electrical signaling in cells and also aid in depolarization of membrane potential through the flow of sodium ions into the cells and their overall function is to maintain the membrane excitability of the cells. The chemical insecticides such as pyrethroids and DDT target the pest by inhibiting the channel 
inactivation while causing channel opening for a prolonged period of time [6].

Limitations of using chemical insecticides Unfortunately, most of the arthropods pests including mosquitoes have developed resistance against the commonly used chemical insecticides. There are four main mechanisms through which these pests have developed resistance. The first one is $k d r$ mediated target site insensitivity which is also known as knockdown resistance. This type of resistance is specifically identified in mosquitoes, which is caused by the mutations in Voltage-gated Sodium Channel (VGSC) genes. So far, there are 11 VGSC mutations identified in the mosquitoes only. The second mechanism is the enhanced metabolic detoxification which results in the detoxification of chemical insecticides through various metabolic activities. There is another drawback of this mechanism of resistance as it causes cross-resistance among different chemical insecticides which have similar chemical structures. For instance, the mosquito populations which became resistant to DDT by adapting the mechanism of enhanced metabolic detoxification also became resistant to deltamethrin due to crossresistance. The third mechanism is the development of a thick cuticle layer which reduces the penetration of insecticide in the body. The vector of malaria Anopheles gambiaehas developed three different proteins, two of which (CPLCG3 and CPLCG4) slows down the penetration while the third (CPF3) is reported to develop desiccation tolerance in the mosquito populations. The last mechanism is mutations in the Glutathion S-tranferase gene (GSTe2) which lead to overexpression of this gene while increasing the metabolism and detoxification of insecticides by the enzyme Glutathion S-transferase enzyme which is naturally present in the mosquitoes [7].

\section{Mosquito control by plant extracts}

The development of different target site and metabolic resistance mechanisms in mosquitoes against chemical pesticides have helped to develop other methods of controlling mosquitoes which have their own advantages and limitations. One such method of controlling mosquitoes at different life stages is the use of plant-based essential oils. The oil extracts of plants Mentha arvensis (Mint), Saussurea lappa (Cactus), Acoruscalamus(Sweet flag), Cymbopogan citrates (Lemongrass) and Ocimum basilicum (Sweet basil) showed significant larvicidal activity against mosquito species Aedesaegypti, the vector of Dengue, Yellow fever, Chikungunya and Zika Virus, and Culex quinquefasciatus, the vector of Lymphatic filariasis. The small concentrations of these plant extracts like Ocimum bacilicum (Sweet basil) are reported to show larvicidal activity against these species [8].

\section{Limitation of plant extracts used as insecticides}

There are certain limitations associated with the use of plant oil extracts for controlling mosquitoes population. First, the extraction of these oils is expensive. Secondly, these oils are effective to be part of only Indoor Residual Sprays (IRS) where mosquitoes reside. They cannot be used on a larger volume of stagnant water [9].

Genetic methods of controlling mosquitoes Due to the limitations of other mosquito management practices, genetic control methods offer a more sophisticated approach. The two most important genetic control methods include the use of the refractory gene and the introduction of lethal gene in the mosquitoes to suppress the mosquito population [10]. Many methods have been used to introduce refractory gene in the mosquito which includes the use of Immunity peptides, Synthetic peptides, one chain antibodies, cell signaling molecules and the 
mechanism of RNA interference. One of the most widely studied methods is Wolbachiabacteria mediated infection to control mosquitoes population. More than $70 \%$ of insects are infected with theseendosymbiotic bacteria. Different strainsofWolbachiahave been used in different parts of the World to control mosquitoes population. Various malariacarrying species of mosquitoes were targeted using different strains of Wolbachia like wAlbB [11]. Use of Wolbachiabacteria for the introduction of a refractory genehas also been proposed.Another method utilizes the introduction of the lethal gene in male mosquitoes when these mosquitoes mate with wild females, they cause the death of females as these females lack the repressor of that gene. This leads to the sex-specific lethality of mosquito populations. There are few other genetic methods which are still under consideration to be applied at commercial levels. These methods include sterile male systems, Self-limiting replacement methods, frequency-dependent gene drive systems, and gene drive systems [12].

\section{Limitations of genetic control methods}

There are many limiting factors that need to be considered before applying a genetic method of controlling disease-transmitting mosquito species. The genetic manipulation of mosquito would need a highly developed molecular laboratory with all the facilities required for the procedures to be undertaken. The genome of the mosquitoes is very complex; therefore, more sophisticated and targeted protocols need to be used to undergo any alteration in the genomic system. These methods are very expensive and what adds to the expense is the need for repeated release of genetically altered mosquito species to maintain the required genetic changes in the population. Another limiting factor is that all species of vector mosquitoes need to be altered separately to control all mosquitoborne diseases which add to the time, labor and expense needed to control all vector species of mosquitoes. Moreover, there are some ethical issues regarding the release of genetically modified mosquitoes in the environment as there could be some unforeseen consequences that may harm the natural diversity of the environment. These are the reasons behind the genetic methods of controlling disease-transmitting mosquito vectors not being much practiced yet [10].

\section{Biological methods of controlling mosquitoes}

After facing the limitations of genetic methods of controlling mosquitoes, the biological methods are more conveniently in use till date. The biological control methods are also known as natural methods. These control methods make use of natural predators, parasites and pathogens of mosquito species to kill them. Many organisms are reported to be the natural pathogens or parasites of mosquitoes. Till now larvivorous fish, entomopathogenic fungi, invertebrate parasites, plant byproducts, and bacteria are being studied to control mosquitoes [13].

\section{Larvivorous fish as natural predators of mosquitoes}

The larvivorous fish are those which feed on the larvae of the mosquitoes and are known to be the natural predators of mosquito larvae. These larvivorous fish were introduced purposely to control mosquito in 1921 in the USA before the introduction of pesticides such as DDT but after the extensive use of effective chemicals, the use of these larvivorous fish was greatly reduced. Since the development of resistance in mosquitoes against chemical pesticides, the use of larvivorous fish to control mosquito is again under consideration. The commonly known and used larvivorous species include Aplocheilus blockii (Dwarf panchax), Aplocheilus lineatus (Malabar Killie), Aphanius dispar (Dispar topminnow), Colisa lalia (Dwarf gourami), Colisa fasciatus 
(Giant gourami), Carassius auratus (Gold fish), Macropodus cupanus (Spiketailed paradise fish), Poecilia reticulate (Guppy) and many others [14].

\section{Limitations of using larvivorous fish as control method}

Tocontrol larvae,there are many criteria which need to be fulfilled for a fish to be suitable enough for its use. These fishes should be small and capable of living in both shallow and deep water with the ability to tolerate drought. These fish should be the natural predator of mosquito larvae while preferring to consume mosquito larvae even in the presence of other food components. These fish should not be used by humans as food so that these can be used only for controlling mosquito. These fish should have the ability to survive the handling and transportation conditions. Since all these criteria are difficult to be fulfilled by a single species of fish, therefore, the use of fish as potent larvicide is limited. Moreover, there are many disadvantages of using larvivorous fish which also limit its use. Some of the larvivorous fish are proved to cause a negative impact on the ecology as these turn out to be opportunistic predators which might consume other zooplanktons in the ecosystem that include other fish and their eggs. To use the larvivorous fish to control mosquitoes, optimal conditions are required to help the fish flourish which is very difficult to be maintained at a constant level. It is also not possible to use these fish in the mosquito habitat where sufficient water is not available for the growth of these fish. All these factors have confined the use of larvivorous fish in only a few habitats and conditions [15].

Entomopathogenic fungi and its toxins as a natural insecticide

Various entomopathogenic fungi are also used for controlling certain species of mosquitoes. The entomopathogenic fungi are those which are pathogenic for the insects. The fungiused for controlling mosquitoes generally alter the physiology of the mosquito and decreases the ability of the mosquito to feed on blood. In the result of this change in physiology and feeding habit, the frequency of disease transmission is greatly reduced [16]. The important fungal species which are entomopathogenic in nature include Beauveria bassiana, Metarhizium anisopliae, Pythium spp, Verticillium lecanii, Leptolegnia chapmani, Lagenidium giganteum, Crypticola spp, Paecilomyces while most important being Baeuveria bassianaa ndMetarhizium anisopliae [17]. Some of these fungal species have been tested in the laboratory against the larvae of Aedes aegypti, Aedes albopictus and Culex quinquefasciatus. Among all these species, Metarhizium anisopliae showed maximum activity against Aedes albopictus [18].

Limitations of using entomopathogenic insecticides

There are certain limitations in the commercialization and large scale production of entomopathogenic fungi. There are certain mycotoxins produced by these fungi which can be harmful not only for plants but also for human beings. These toxins inhibit certain biochemical reactions in the body and may prove to be cytotoxic and immunosuppressive. Infections were reported in normal individuals and immunecompromised patients who came in contact with commercially available entomopathogenic fungi [19]. The large scale manufacturing of entomopathogenic fungi is not only expensive but can be considered a challenge as the fungal spores are unstable and have a short shelf life. Moreover, most of the fungal spores and species are active only in water but still take a long time to kill the insects or mosquito larvae. The studies and researches done on the usage of these entomopathogenic fungi have not helped to successfully make a product on a large scale. The commercialization of entomopathogenic 
fungi is still confined to control crop pests [20].

\section{Use of invertebrates as natural predators of mosquitoes}

\section{Use of predator giant mosquitoes}

Invertebrates which are the natural predators of mosquitoes and feed on them provide another biological control remedy. These invertebrates include the elephant mosquito Toxorynchites spp. and freshwater crustacean cyclopoid copepods [21]. Toxorynchites splendens is the most effective and widely reported species among the other mosquito predator species [22]. They naturally feed on the larvae of other mosquito species including that of Culex, Aedes, and Anopheles which are the major disease transmitter genera of mosquitoes. The advantage of using Toxorynchites spp. as the natural predator of these larvae is that the female members of this genus do not feed on human blood. Therefore, there is no chance of this predating mosquito becoming a vector of human diseases. There is no reported harm of this mosquito on human beings. This naturally predating mosquito is able to feed on more than 10 mosquito larvae a day while being capable of consuming around 5000 mosquito larvae in its whole life cycle which make a great deal for controlling the diseasetransmitting mosquitoes [23].

\section{Limitation of using predator giant mosquito}

Before considering the use of Toxorynchites mosquitoes as the perfect and most effective natural method of controlling the diseasetransmitting mosquitoes, there are some factors and limitations which need to be considered. The female Toxorynchites mosquitoes do not oviposit or lay eggs in the waters where Aedes aegyptiis present due to the presence of pheromones present in the breeding habitat of Aedes mosquito. This can lead to the eventual loss of Toxorynchites population in the water infested with Aedes mosquitoes. Moreover, a large population of
Toxorynchites is required to prey on a handsome number of vector mosquitoes. Another issue of the use of this predator is that this control method cannot be used in combination with other methods to increase the effectiveness as all other methods or insecticides would kill both the predator and the prey [24].

\section{Use of cyclopoid as natural predators of mosquitoes}

The other natural invertebrate predators of mosquitoes are cyclopoid copepods with the most effective genus being Macrocyclops.Cyclopoid copepods are small crustaceans that inhabit lakes, marine water, rivers, humid rocks, ponds and other water bodies [25]. Mesocyclops and Macrocyclops species mainlyfeed on Aedessppmosquitoes first larval instar. This method was proved successful in regions including French Polynesia and Queensland, Australia. In Vietnam,a large scale study was conducted to reduce the incidence of Dengue virus by reducing the population of mosquitoes using Mesocyclops and health education.The method was proved effective in lowering the number of mosquitoes population in that particular area. [16]. Some studies reported that one copepod is capable of consuming more than 40 larvae a day [26] which can prove to be the most effective natural method of controlling these disease-transmitting vector mosquitoes.

\section{Limitations of using Cyclopoid copepods}

Similar to the other natural or biological control methods, the use of cyclopoid copepods also has some limitations and disadvantages. Studies have suggested that these copepods have been most effective against Aedeslarvae but these copepods have very little effect on Culexand Anopheles larvae which has limited its use on large scale [21]. Moreover, to rear these crustaceans, an adequate amount of food and water would be required in the mosquito infected medium. The food and water provided to these 
crustaceans may help the mosquitoes to flourish which is notagainst the whole idea of controlling mosquitoes. There is no negative impact of these predators on the natural environment but there can be some serious health impacts on humans which might lead to an epidemic situation. These copepods have been known to be a vector of guinea worm Dracunculus medinensis which is a human parasite. Moreover, the copepods can be involved in the transmission of other diseases such as cholera if they are introduced in the drinking water [27]. Since these copepods can cause epidemics, introducing these diseases while controlling the mosquito-borne diseases would not be a suitable option.

\section{Use of Bacillus sphaericusas natural insecticide}

One suitable option for controlling the mosquito vectors is the use of bacteria which are pathogenic to the mosquito species. One such bacterium is Bacillus sphaericus (Bs) which is gram-positive, strict aerobe bacteria that cannot use sugars as carbon source as it lacks the ability to ferment sugars. Bs is the natural inhabitant of soil and aquatic environment from where it can be easily isolated [28] Bacillus spearichus has been reported to control Culex spp. and some of the species of genus Anopheles. The Bs is an endospore former and produces a parasporal protein which, upon ingestion by the larvae, gets hydrolyzed in the stomach by the action of proteases. This solubilized protein damages both the nervous and digestive system and often lead to septicemia. The whole mechanism takes 48 hours to kill the mosquito larvae that have ingested this toxic protein [29].

\section{Limitations}

Unfortunately, the use of Bacillus sphaericus did not turn out as the best method of controlling mosquitoes. The bacteria were reported to be effective against Culex and Anopheles but the Aedeslarvae are insensitive to the toxic protein produced by Bacillus sphearicus which means that it cannot be used to control certain diseases such as Dengue Fever and Chikungunya. Besides, since there is only one toxic protein produced by these bacteria, there are chances that mosquitoes might develop resistance against this protein [21]. Therefore, Bacillus sphearicus has found limited use as a biocontrol agent against mosquito larvae.

\section{Wolbachia as natural pathogen of mosquitoes}

The use of one other bacterium to control the disease-transmitting mosquitoes has been explained in the genetic control methods. Wolbachia is an alphaprotobacterium which is a natural pathogen and intracellular parasite of various insect species. These bacteria are known to reside in the cytoplasm of the host cells and have the ability to be transmitted both horizontally and vertically [30]. It has been predicted that Wolbachia is present naturally in almost $60 \%$ species of insects in which Aedesis not included. It has been suggested that using Wolbachia as biocontrol strategy can reduce or eliminate the incidence of viral diseases Dengue, Chikungunya and transmission ofzika virus [31]. The Wolbachia infects the cells of the host and cause protection against various RNA viruses. This is the reason that the transmission of viruses is greatly reduced as the bacteria are transmitted maternally through the infected mosquitoes. Aedesaegyptihas been artificially infected by Wolbachiato inhibit the replication of a virus in the mosquito. The same principle is being applied to control other mosquito species from transmitting other viral diseases [32]. Researchers from the United States have studied the genome of Wolbachiamobilome. They found a putative plasmid called $\mathrm{pWCP}$ (Plasmid of Wolbachia in Culexpipiens ) for the first time in this bacterium which could be used for future genetic manipulation for enhancing its infection efficacy. This 
discovery could lead to effective containment of Zika and dengue virus in the future [33].

\section{Limitation}

There might be some issues which need to be addressed before using this method of controlling mosquitoes. First is the fact that Wolbachia has the antiviral activity that can inhibit the replication of RNA virus, so only viral infections can be controlled by using these bacteria. Since Malaria is caused by plasmodium, which is a parasite or protozoa, so this strategy is of no use in case of Malarial Management. Secondly, this control strategy is not sustainable enough to be applied only once. Repeated release of artificially infected mosquitoes is required to completely control the transmission for which extensive studies regarding the mosquito population in a locality is required. Thirdly, Wolbachia is not transmitted to humans by mosquito bites, which is an advantage, but it might get transmitted to other living organisms present in the environment which are natural predators of mosquitoes. This can disturb the natural diversity of the ecosystem [34].

\section{Conclusion}

The chemicals used to kill mosquitoes and other insects and pests have already damaged our environment and health.Also,repetitive utilization of these chemicals has developed resistance in mosquitoes which is taking control of these vectors more difficult.Therefore, more sophisticated and effective methods are required to be introduced to kill mosquitoes without harming natural diversity of the ecosystem as well as the non-targeted organisms. The review presented some of the approaches that can help to build sustainable vector control strategies for minimizing the spread of diseases. Each biological method has its advantages and limitations. However various factors like demography, ecology, education, and suitability of treatment and many other factors need to be considered before adopting any of the methods. Continuous research for improving effectiveness these environmentally friendly alternatives will be beneficial for long term control of the mosquito population.

\section{Authors' contributions}

Conceived the idea: Rasheda Jabeen, Wrote the paper: A Chaudhry, R Jabeen, B Sarfraz \& S Mazhar, Corresponding author: S Mazhar

\section{References}

1. WHO (2016). Mosquito-borne diseases, World Health Organization.

2. CDC (2018). Mosquito-Borne Diseases, The National Institute for Occupational Safety and Health (NIOSH).

3. Cui F, Raymond M \&Qiao CL (2006). Insecticide resistance in vector mosquitoes in China. Pest Manag Sci 62: 1013-1022.

4. Poupardin R, Reynaud S, Strode C, Ranson H, Vontas J \& David JP (2008). Cross-induction of detoxification genes by environmental xenobiotics and insecticides in the mosquito Aedes aegypti: impact on larval tolerance to chemical insecticides. Insect Biochem Mol Biol 38: 540-551.

5. WHO (2014). A global beief on vector borne diseases. World Health Organization.

6. Silver KS, Du Y, Nomura Y, Oliveira EE, Salgado VL, Zhorov BS \& Dong K (2014). Voltage-gated sodium channels as insecticide targets. Adv In Insect Phys 46: 389-433.

7. Jugulam M, Niehues K, Godar AS, Koo DH, Danilova T, Friebe B, Sehgal S, Varanasi VK, Wiersma A \& Westra P (2014). Tandem amplification of a chromosomal segment harboring 5enolpyruvylshikimate-3-phosphate synthase locus confers glyphosate resistance in Kochia scoparia. Plant Physiol 166: 1200-1207.

8. Soonwera M \&Phasomkusolsil S (2015). Efficacy of Thai herbal essential oils as 
green repellent against mosquito vectors. Acta Trop142: 127-130.

9. Mustapha O (2017). The effect of terpenes on the life cycle of the malaria parasite.Msc thesis. Deptt of med, Univ of Witwatersrand, Johannesburg. Johannesburg, South Africa.

10. Wilke ABB \& Marrelli MT (2012). Genetic control of mosquitoes: population suppression strategies. Rev Inst Med Trop Sao Paulo 54: 287-292.

11. El Hadji AN, Bassene H, Fenollar F \& Mediannikov O (2018). Biological Control of Mosquito-Borne Diseases: The Potential of Wolbachia-Based Interventions in an IVM Framework. $J$ of Tropical Med 2018: 1-15.

12. Alphey L (2014). Genetic control of mosquitoes. Annu Rev Entomol 59: 204210.

13. Rodríguez-Pérez MA, Howard AF \& Reyes-Villanueva F(2012). Biological control of dengue vectors. 2nd Ed. InTech; Mexico, pp 32.

14. Walshe DP, Garner P, Adeel AA, Pyke GH \& Burkot TR (2017). Larvivorous fish for preventing malaria transmission. CDSR (12): 44-52.

15. Chandra G, Bhattacharjee I, Chatterjee $S$ \&Ghosh A (2008). Mosquito control by larvivorous fish. Indian J Med Res 127: 13-28.

16. Huang YJ, Higgs $S \&$ Vanlandingham D (2017). Biological control strategies for mosquito vectors of arboviruses. Insects 8: 21-29.

17. El-Ghany A, Roushdy M \& Mohamed A (2015). Efficacy of certain plant extracts as safe fungicides against phytopathogenic and mycotoxigenic fungi. Agric Biol Sci J 1: 71-75.

18. Lee SJ, Kim S, Yu JS, Kim JC, Nai Y-S \& Kim JS (2015). Biological control of Asian tiger mosquito, Aedes albopictus (Diptera: Culicidae) using Metarhizium anisopliae JEF-003 millet grain. J Asia Pac Entomol 18: 217-221.

19. Hu Q, Li F \& Zhang Y (2016). Risks of mycotoxins from mycoinsecticides to humans. Biomed Res Int 7: 95-101.

20. Nahusenay DG \&Abate GA (2018). Evaluation of selected botanical aqueous extracts against cabbage aphid (Brevicoryne brassicae L. (Hemiptera: Aphididae) on cabbage (Brassicae oleraceae L.) under field condition in Kobo District, North Wollo, Ethiopia. Ethiopia J Hortic For 10: 69-78.

21. Mazzacano C \&Black SH (2013). Ecologically sound mosquito management in wetlands. An overview of mosquito control practices, the risks, benefits, and nontarget impacts, and recommendations on effective practices that control mosquitoes, reduce pesticide use, and protect wetlands. The Xerces Society for Invertebrate Conservation, Portland, Oregon.

22. Zuharah WF, Fadzly N, Yusof NA \&Dieng H (2015). Risky behaviors: effects of Toxorhynchites splendens (Diptera: Culicidae) predator on the behavior of three mosquito species.J. Insect Sci 15: 35-43.

23. Faithpraise F, Idung J, Usibe B, Chatwin C, Young R \& Birch P (2014). Natural control of the mosquito population via Odonata and Toxorhynchites. IJIRSET 3: 12898-12911.

24. Ong SQ (2016). Dengue vector control in Malaysia: A review for current and alternative strategies. SAINS MALAYS 45: 777-785.

25. Perbiche-Neves G, da Rocha CE \& Nogueira MG (2014). Estimating cyclopoid copepod species richness and geographical distribution (Crustacea) across a large hydrographical basin: comparing between samples from water column (plankton) and macrophyte stands. Zoologia (Curitiba) 31: 239-244. 
26. Sarwar M (2015). Elimination of Dengue by Control of Aedes Vector Mosquitoes (Diptera: Culicidae) Utilizing Copepods (Copepoda: Cyclopidae). Ijbbe 1: 53-58.

27. Cardôso HCB, da Silva BQ, de Assis TB \& Lopez LCS (2013). Effects of predation by the copepod Mesocyclops ogunnus on the sex ratios of mosquito Aedes albopictus. Hydrobiologia 705: 55-61.

28. Luna-Finkler CL \& Finkler L (2012). Bacillus sphaericus and Bacillus thuringiensis to insect control: Process development of small scale production to pilot-plant-fermenters.

29. Thi S \&Abidha S (2010). Mosquitocidal bacterial toxins (Bacillus sphaericus and Bacillus thuringiensis serovar israelensis): Mode of action, cytopathological effects and mechanism of resistance. J Physiol Pathophysiol 1: 22-38.
30. Johnson K (2015). The impact of Wolbachia on virus infection in mosquitoes. Viruses 7: 5705-5717.

31. Soares AA (2016). Promising new tools to fight Aedes mosquitoes. Bull. World Health Organ 94: 562563.

32. Jeffries CL \& Walker $T$ (2015) The potential use of Wolbachia-based mosquito biocontrol strategies for Japanese encephalitis. PLoS Negl Trop Dis 9: 76-82.

33. Julie SRB, Cruaud C, Shaiber A, Esen O, Weill M, Makoundou P, Lolans K, Andrea R. Watson, I, Seth R. \& Eren A (2019). The Wolbachia mobilome in Culex pipiens includes a putative plasmid. Nat Commun 10: 45-49.

34. Rainey SM, Shah P, Kohl A \& Dietrich I (2014). Understanding the Wolbachiamediated inhibition of arboviruses in mosquitoes: progress and challenges. $J$ Gen Virol 95: 517-530. 